\title{
JQF: Coverage-Guided Property-Based Testing in Java
}

\author{
Rohan Padhye \\ University of California, Berkeley \\ USA \\ rohanpadhye@cs.berkeley.edu
}

\author{
Caroline Lemieux \\ University of California, Berkeley \\ USA \\ clemieux@cs.berkeley.edu
}

\author{
Koushik Sen \\ University of California, Berkeley \\ USA \\ ksen@cs.berkeley.edu
}

\begin{abstract}
We present JQF, a platform for performing coverage-guided fuzz testing in Java. JQF is designed both for practitioners, who wish to find bugs in Java programs, as well as for researchers, who wish to implement new fuzzing algorithms.

Practitioners write QuickCheck-style test methods that take inputs as formal parameters. JQF instruments the test program's bytecode and continuously executes tests using inputs that are generated in a coverage-guided fuzzing loop. JQF's input-generation mechanism is extensible. Researchers can implement custom fuzzing algorithms by extending JQF's Guidance interface. A Guidance instance responds to code coverage events generated during the execution of a test case, such as function calls and conditional jumps, and provides the next input. We describe several guidances that currently ship with JQF, such as: semantic fuzzing with Zest, binary fuzzing with AFL, and complexity fuzzing with PerfFuzz.

JQF is a mature tool that is open-source and publicly available. At the time of writing, JQF has been successful in discovering 42 previously unknown bugs in widely used open-source software such as OpenJDK, Apache Commons, and the Google Closure Compiler.
\end{abstract}

\section{CCS CONCEPTS}

- Software and its engineering $\rightarrow$ Software testing and debugging.

\section{KEYWORDS}

Coverage-guided fuzzing, property-based testing, QuickCheck

\section{ACM Reference Format:}

Rohan Padhye, Caroline Lemieux, and Koushik Sen. 2019. JQF: CoverageGuided Property-Based Testing in Java. In Proceedings of the 28th ACM SIGSOFT International Symposium on Software Testing and Analysis (ISSTA '19), fuly 15-19, 2019, Beijing, China. ACM, New York, NY, USA, 4 pages. https://doi.org/10.1145/3293882.3339002

\section{INTRODUCTION}

Coverage-guided fuzzing (CGF) has recently become a very popular technique for automatic test-input generation. CGF tools like AFL [17] and libFuzzer [7] have discovered thousands of bugs and security vulnerabilities in programs that parse binary data, such as

Permission to make digital or hard copies of all or part of this work for personal or classroom use is granted without fee provided that copies are not made or distributed for profit or commercial advantage and that copies bear this notice and the full citation on the first page. Copyrights for components of this work owned by others than the author(s) must be honored. Abstracting with credit is permitted. To copy otherwise, or republish, to post on servers or to redistribute to lists, requires prior specific permission and/or a fee. Request permissions from permissions@acm.org.

ISSTA '19, fuly 15-19, 2019, Beijing, China

(C) 2019 Copyright held by the owner/author(s). Publication rights licensed to ACM. ACM ISBN 978-1-4503-6224-5/19/07 ..\$15.00

https://doi.org/10.1145/3293882.3339002

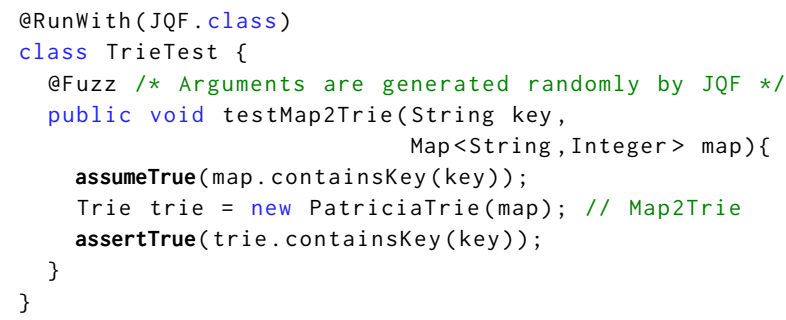

Figure 1: A sample property test using JQF that checks the construction of a Trie data structure in Apache Commons from an input JDK Map. Fires an assertion violation (bug COLLECTIONS-714) when fuzzing with ZestGuidance.

image decoders and media players. CGF works by first inserting lightweight instrumentation in a program under test for collecting code coverage. Then, the program is continuously executed with randomly generated inputs. If the program crashes, a bug is found. Instead of generating inputs from scratch, CGF evolves a set of saved inputs, starting with user-provided seed inputs. New inputs are generated via byte-level mutations such as bit flips and random insertion of interesting values. If a new input leads to an increase in code coverage, it is saved for subsequent mutation. The process repeats until a time budget expires.

The research community has produced several extensions to the basic CGF algorithm. Manès et al. [11] provide an extensive survey of various custom fuzzing algorithms found in the literature. For each of these new fuzzing algorithms, researchers produced standalone variants of AFL, libFuzzer, or other fuzzing tools.

In spite of all this research effort, most existing fuzzing tools target $\mathrm{x} 86$ binaries or $\mathrm{C} / \mathrm{C}++$ programs that expect inputs as binary or textual files. These tools are not suited for driving conventional software tests, where inputs can be arbitrary data structures. Property-based testing tools in the lineage of QuickCheck [3] allow randomized testing of such highly structured inputs, but don't support a feedback-directed fuzzing loop.

We present JQF, a platform for performing coverage-guided fuzzing of Java programs. The JQF platform meets the following design goals:

(1) JQF enables practitioners to use coverage-guided fuzzing for testing programs that require structured inputs, using the familiar style of property-based testing.

(2) JQF enables researchers to prototype new coverage-guided fuzzing algorithms to drive property-based tests.

JQF is publicly available at https://github.com/rohanpadhye/jqf as well as on Maven central (edu. berkeley.cs. jqf: jqf-fuzz). 


\section{FUZZING WITH JQF}

Practitioners can use JQF to automatically generate test inputs for parameterized test methods using coverage-guided fuzzing. Figure 1 shows an example of a JQF test driver written in Java, which aims to check a basic property of the class PatriciaTrie from Apache Commons Collections. A trie data structure can be constructed from a pre-existing mapping of strings in a JDK Map object.

The test method testMap2Trie checks the following property: Given an arbitrary string key and a JDK map whose keys are strings, if key exists within map, then a trie constructed from this map should also contain the same key. The @Fuzz annotation on the test method enables JQF to automatically generate random instances of map and key to verify this property. The JUnit Assume API allows the user to specify preconditions on the generated inputs (e.g. Line 6). Test generation can be launched via JQF's Apache Maven plugin ${ }^{1}$ :

mvn jqf:fuzz -Dclass=TrieTest -Dmethod=testMap2Trie

By default, JQF uses the Zest algorithm (§4.2) to generate test inputs. Fuzzing continues either until it is explicitly stopped, until a userspecified timeout expires, or until a test failure is encountered.

For the test in Figure 1, the Zest fuzzing engine often finds a test failure in about 5 seconds, after executing about 5,000 test inputs (of which over 1,700 satisfy the precondition on Line 6). The failing test case leads to an assertion violation at Line 8 due to a very special corner case, which reveals a bug in Apache Commons Collections v4.3. If the input map contains two distinct keys that differ only in a trailing null character, say " $x$ " and " $x \backslash u 0000 "$ ", then the trie cannot distinguish between them and ends up storing only one of the two keys. If the input key is also " $x$ ", then the bug is revealed 2 .

JQF was specifically designed to enable practitioners to write test methods in the familiar style of property-based testing. JQF is built on top of junit-quickcheck [6], which itself is a Java port of the popular QuickCheck [3] tool. Thus, the test driver in Figure 1 can still be run with vanilla junit-quickcheck, which randomly generates test inputs without using code coverage feedback. However, random-from-scratch input generation is exceedingly unlikely to generate inputs fitting precise bug-revealing conditions, like those described above. Pure random generation does not find a failing test case for Figure 1 even after 30 minutes (over 7 million executions).

\section{FUZZING FRAMEWORK}

We next explain how JQF generates random inputs, such as map and key in Figure 1, using coverage-guided algorithms called guidances.

\subsection{The Guidance Interface}

Figure 2 shows the Guidance interface. Researchers can implement this interface to specify a coverage-guided fuzzing algorithm. Guidance instances are stateful objects whose methods are invoked by the JQF framework in a fuzzing loop (depicted in Figure 3).

The Guidance method hasInput () returns whether a new input is available; the return value false ends fuzzing. The getInput() method returns the next input generated by the Guidance, as an InputStream. This stream is used to generate structured inputs such as Map objects (see §3.2). The structured inputs, called args in

\footnotetext{
${ }^{1}$ Non-Maven users can launch JQF programatically or via command-line scripts

${ }^{2}$ We stumbled upon this bug while writing an example for this paper.
}

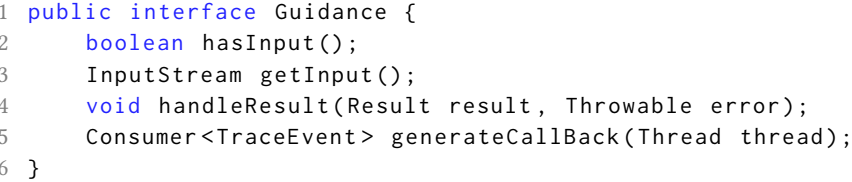

Figure 2: The Guidance interface provided by JQF.

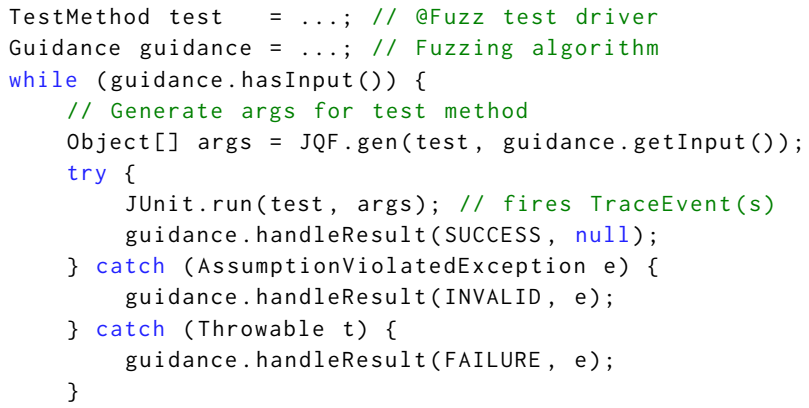

Figure 3: Pseudo-code of the fuzzing loop.

Figure 3, are then used to execute the test method via JUnit (Line 7). Test execution generates TraceEvents, whose handling is described in §3.3. At the end of test execution, the Guidance . handleResult () method is invoked. The result can either be SUCCESS, INVALID, or FAILURE, depending on whether the test method returned normally (Line 8), due to a violation of assume (Line 10), or due to an exception/assertion violation (Line 12), respectively. The Guidance instance updates its internal state based on the handling of code coverage events and the test result. The internal state is then used to generate new inputs in subsequent iterations of the fuzzing loop.

\subsection{Parametric Generators}

The arguments to a test method-such as map and key in Figure 1-are generated using the same mechanisms as supported by junit-quickcheck. In general, inputs of type T are generated by a backing Generator $\langle\mathrm{T}\rangle$, which provides a method to randomly sample a new instance of T. junit-quickcheck can either (1) implicitly pick a suitable generator from a library that it provides, (2) be directed to synthesize such a generator automatically, e.g. using the constructors or public fields of class T, or (3) be provided with a hand-written Generator $\langle T\rangle$.

In all cases, the generator uses a Source0fRandomness object, which provides an API for making non-deterministic decisions such as: choosing from a list of alternatives (e.g. whether to instantiate a TreeMap or HashMap for map in Figure 1), picking random sizes (e.g. how many entries to insert in map), or populating primitives (e.g. what keys and values to insert in map). In junit-quickcheck, the default Source0fRandomness is backed a pseudo-random stream of bytes. JQF overrides this source to use the stream returned by Guidance.getInput() instead (ref. Line 5 in Figure 3), thereby making the generators deterministically dependent on the guidance. 


\subsection{Code Coverage Events}

When coverage-guided fuzzing is launched (e.g. via mvn jqf: fuzz), the test program's classes are instrumented on-the-fly using the ASM bytecode manipulation library [13]. The instrumentation adds logic to generate TraceEvents during test execution. For example, a BranchEvent is generated when a test program executes a conditional branch, a CallEvent accompanies a method invocation, and an AllocEvent signals the creation of a new object or array on the heap. These event objects contain information about their source program locations as well other event-specific data. When a trace event $e$ is generated in thread $t$, JQF invokes the function handle_t $(e)$, where handle_t is the callback returned by Guidance.generateCallBack $(t)$. The guidance must choose how to update its internal state based on this coverage information, which will presumably be used to generate subsequent inputs.

\section{GUIDANCES}

JQF currently ships with the following Guidance implementations.

\subsection{No Guidance}

The most trivial guidance, called NoGuidance, returns an infinite stream of random values every time getInput() is called. This guidance completely ignores code coverage events. This guidance is almost equivalent to using vanilla junit-quickcheck.

\subsection{Zest Guidance}

JQF's default guidance implements the Zest algorithm [15], which is specifically designed for coverage-guided property testing. The ZestGuidance returns dynamically sized parameter sequences via the getInput () method, which are generated randomly for the first iteration of the fuzzing loop. Zest maintains a set of saved parameter sequences. The ZestGuidance generates new inputs by randomly mutating previously saved parameter sequences. Bytelevel mutations on these parameter sequences correspond to structural mutations in the generated test inputs. For example, a random mutation in the parameter sequence for map in Figure 1 may lead to the corresponding Generator $<$ Map $>$ to produce the next map with an additional entry. Dynamic sizing allows the parameter sequences to be lazily extended (if the Generator needs to make more choices than expected) or to be efficiently truncated (if the Generator makes fewer choices). Further, Zest separately tracks code coverage achieved by all test executions and code coverage by valid test executions (i.e., those whose result is SUCCESS). If a mutated parameter sequence leads to new code coverage overall, or if it leads to a valid test that covers code which has not been covered by any previous valid test, then the sequence is saved for subsequent mutation. Zest has been used to find complex semantic bugs, such as issues within compiler optimizations ${ }^{3}$.

\subsection{AFL Guidance}

JQF supports input generation using the popular AFL [17] tool, unmodified. This is possible because AFL, which is designed to fuzz $\mathrm{C} / \mathrm{C}++$ programs and $\mathrm{x} 86$ binaries, communicates with instrumented test programs via inter-process messages and a code

\footnotetext{
${ }^{3}$ https://github.com/google/closure-compiler/issues/2842
}

coverage map in shared memory. The AFLGuidance in JQF implements this communication protocol via a proxy program. The proxy mocks an AFL-instrumented test target that reads input from a specific file. AFLGuidance. getInput() simply returns the contents of this file, which is continuously updated by AFL. During test execution, AFLGuidance collects code coverage information by handling TraveEvents. When AFLGuidance.handleResult() is invoked, the coverage information is written to AFL's shared memory region via the proxy. Calls to AFLGuidance. hasInput() block until AFL is ready with the next input.

AFL's mutation strategy uses various heuristics that are applicable to programs that parse fixed-size binary files (e.g. media players). Further, AFL does not explicitly distinguish between INVALID and FAILURE results. Due to these reasons, JQF's AFLGuidance is most effective when used with test methods that take only one argument of type InputStream (since Generator<InputStream $>$ returns the guidance-generated input stream as-is), and that do not use any assume statements. For example, AFLGuidance has been used to fuzz OpenJDK's ImageIO library that reads PNG and JPEG files ${ }^{4}$, as well as Apache PDFBox's processing of PDF documents ${ }^{5}$.

\subsection{PerfFuzz Guidance}

PerfFuzz [10] is a technique for automatically generating test inputs that maximize performance counters, such as loop execution counts. PerfFuzz's goal is to automatically discover hot spots and performance bottlenecks. PerfFuzz is a fork of AFL that extends its code coverage map with performance feedback in the form of $\langle k, v\rangle$ pairs where $v$ is a value to be maximized for every key $k$. PerfFuzz saves a mutated input either if it leads to new code coverage, or if it maximizes the value of $v$ for some key $k$.

JQF's PerfFuzzGuidance is a sub-class of AFLGuidance which overrides handleResult() to communicate this additional performance map via the proxy program. PerfFuzzGuidance can be configured either to find hot spots (where keys are branch locations and values are execution counts for the corresponding branch) or to find memory consumption issues (where keys are allocation sites and values are number of bytes allocated at the corresponding site). For example, we used PerfFuzzGuidance to find an algorithmic complexity bug in the Google Closure Compiler, where reporting a specific case of syntax error in a JavaScript program can take time that is exponential in the size of the input program ${ }^{6}$. With the memory allocation feedback, we found an issue in OpenJDK's handling of PNG images that specify very large dimensions ${ }^{7}$.

\subsection{Repro Guidance}

Finally, the ReproGuidance is a trivial guidance whose getInput() method returns the contents of a given file on disk, and then ends the loop. This guidance enables debugging of saved test failures.

\section{EVALUATION AND IMPACT}

Table 1 summarizes the impact that JQF has had in discovering previously unknown bugs in widely used Java software. These

\footnotetext{
${ }^{4}$ https://bugs.openjdk.java.net/browse/JDK-8191073

${ }^{5} \mathrm{https}$ //issues.apache.org/jira/browse/PDFBOX-4333

${ }^{6}$ https://github.com/google/closure-compiler/issues/3173

${ }^{7}$ https://bugs.openjdk.java.net/browse/JDK-8190332
} 
Table 1: Number of new bugs discovered using JQF.

\begin{tabular}{lcc}
\hline Project & Bugs Found & Bugs Fixed \\
\hline OpenJDK - ImageIO & 9 & 9 \\
OpenJDK - DateTime & 2 & 1 \\
Apache Commons - Lang & 1 & 1 \\
Apache Commons - Compress & 2 & 2 \\
Apache Commons - Collections & 1 & 0 \\
Apache Maven & 3 & 3 \\
Apache Ant & 1 & 1 \\
Apache BCEL & 8 & 0 \\
Apache PDFBox & 4 & 4 \\
Apache Tika & 2 & 2 \\
Google Closure Compiler & 4 & 1 \\
Mozilla Rhino & 5 & 0 \\
\hline Total & 42 & 24 \\
\hline
\end{tabular}

bugs were found over the course of various experiments performed throughout 2017-2019. The Zest paper [15] describes a systematic study involving five of the projects from this table and three different guidances. The study showed that NoGuidance is not very reliable, that AFLGuidance is effective in finding bugs in syntax parsers, and that ZestGuidance excels at finding semantic bugs. This study resulted in the discovery of 20 of the bugs in Table 1.

Of the total 42 bugs found using JQF, 11 semantic bugs were found with ZestGuidance, 29 syntax parsing bugs were found with AFLGuidance, and 2 bugs were found with PerfFuzzGuidance. 24 of the 42 reported bugs have been fixed at the time of writing, while the rest await patches.

Notably, 7 of the 42 bugs (including 4 security vulnerabilities with assigned CVEs) were discovered by two independent practitioners who are not affiliated with the authors of this paper. We were made aware of JQF's success via social media [1] and blog posts [12]. All 7 of these bugs have been fixed. We are encouraged by these findings, and believe that they provide evidence to support JQF's usefulness to the software testing community at large.

\section{RELATED WORK}

JQF is one of few tools that enable coverage-guided fuzzing of Java programs. Kelinci [9] is a wrapper around afl-fuzz that targets Java programs. Unlike JQF's extensible guidance mechanism, Kelinci's instrumentation directly updates AFL-specific coverage feedback; therefore, it cannot easily be adapted to work with tools like PerfFuzz. Kelinci also expects a test driver with a main method that reads inputs as files, in contrast to JQF's property-based testing approach. Thus, Kelinci does not support structured input fuzzing algorithms such as Zest. Further comparison between Kelinci and JQF can be found in a blog post by an independent security company [12]. Barro [8] has implemented another wrapper around AFL for fuzzing Java main programs, similar to Kelinci. This tool actually borrows its dynamic instrumentation logic from JQF itself.

There exist several other tools for generating tests for Java programs. Randoop [14] generates method call sequences randomly, but does not use code coverage feedback. Evosuite [4] evolves a test suite using genetic algorithms. Java PathFinder [16] enables symbolic execution of Java programs. Korat [2] systematically explores the input space of Java predicates. UDITA [5] performs bounded exploration of test-input generators. Our tool, JQF, differs mainly in that supports a different mechanism for test-input generation: that of coverage-guided fuzzing.

\section{ACKNOWLEDGMENTS}

We would like to thank Tobias Ospelt of modzero AG for reporting various issues with alpha versions of JQF. We appreciate the feedback of Tim Allison, VP of Apache Tika, on early bugs found by JQF. We are also grateful to Paul Holser for developing the very extensible junit-quickcheck library, using which JQF is built. This research is supported in part by gifts from Samsung, Facebook, and Futurewei, and by NSF grants CCF-1409872 and CNS-1817122.

\section{REFERENCES}

[1] Tim Allison. 2018. \#threeCheersForFuzzing. https://twitter.com/_tallison/status/ 1050455776848949249. Accessed April 17, 2019.

[2] Chandrasekhar Boyapati, Sarfraz Khurshid, and Darko Marinov. 2002. Korat: Automated Testing Based on Java Predicates. In Proceedings of the 2002 ACM SIGSOFT International Symposium on Software Testing and Analysis (ISSTA '02). ACM, New York, NY, USA, 123-133. https://doi.org/10.1145/566172.566191

[3] Koen Claessen and John Hughes. 2000. QuickCheck: A Lightweight Tool for Random Testing of Haskell Programs. In Proceedings of the 5th ACM SIGPLAN International Conference on Functional Programming (ICFP).

[4] Gordon Fraser and Andrea Arcuri. 2011. EvoSuite: Automatic Test Suite Generation for Object-oriented Software. In Proceedings of the 19th ACM SIGSOFT Symposium and the 13th European Conference on Foundations of Software Engineering (ESEC/FSE'11).

[5] Milos Gligoric, Tihomir Gvero, Vilas Jagannath, Sarfraz Khurshid, Viktor Kuncak, and Darko Marinov. 2010. Test generation through programming in UDITA. In Proceedings of the 32nd ACM/IEEE International Conference on Software Engineering - Volume 1, ICSE 2010, Cape Town, South Africa, 1-8 May 2010. 225-234. https://doi.org/10.1145/1806799.1806835

[6] Paul Holser. 2014. junit-quickcheck: Property-based testing, JUnit-style. https: //pholser.github.io/junit-quickcheck. Accessed January 11, 2019.

[7] LLVM Compiler Infrastructure. 2016. libFuzzer. https://llvm.org/docs/LibFuzzer. html. Accessed April 17, 2019.

[8] Jussi Judin. 2018. Binary rewriting approach [...] to fuzz Java applications with afl-fuzz. https://github.com/Barro/java-afl. Accessed April 17, 2019.

[9] Rody Kersten, Kasper Luckow, and Corina S Păsăreanu. 2017. POSTER: AFLbased Fuzzing for Java with Kelinci. In Proceedings of the 2017 ACM SIGSAC Conference on Computer and Communications Security. ACM, 2511-2513.

[10] Caroline Lemieux, Rohan Padhye, Koushik Sen, and Dawn Song. 2018. Perffuzz: Automatically Generating Pathological Inputs. In Proceedings of the 27th ACM SIGSOFT International Symposium on Software Testing and Analysis (ISSTA 2018). ACM, New York, NY, USA, 254-265. https://doi.org/10.1145/3213846.3213874

[11] Valentin J. M. Manès, HyungSeok Han, Choongwoo Han, Sang Kil Cha, Manuel Egele, Edward J. Schwartz, and Maverick Woo. 2018. Fuzzing: Art, Science, and Engineering. CoRR abs/1812.00140 (2018). arXiv:1812.00140 http://arxiv.org/abs/ 1812.00140

[12] Tobias Ospelt. 2018. AFL-based Java fuzzers and the Java Security Manager. https://www.modzero.ch/modlog/archives/2018/09/20/java_bugs_with_ and_without_fuzzing/index.html. Accessed April 17, 2019.

[13] OW2 Consortium. 2018. ObjectWeb ASM. https://asm.ow2.io. Accessed August 21, 2018.

[14] Carlos Pacheco and Michael D. Ernst. 2007. Randoop: Feedback-directed Random Testing for Java. In Companion to the 22nd ACM SIGPLAN Conference on Objectoriented Programming Systems and Applications Companion (OOPSLA '07).

[15] Rohan Padhye, Caroline Lemieux, Koushik Sen, Mike Papadakis, and Yves Le Traon. 2019. Semantic Fuzzing with Zest. In Proceedings of the 28th ACM SIGSOFT International Symposium on Software Testing and Analysis (ISSTA '19). https: //doi.org/10.1145/3293882.3330576

[16] Willem Visser, Corina S PÇŐsÇŐreanu, and Sarfraz Khurshid. 2004. Test input generation with Java PathFinder. In ACM SIGSOFT Software Engineering Notes, Vol. 29. ACM, 97-107.

[17] Michał Zalewski. 2014. American Fuzzy Lop. http://camtuf.coredump.cx/afl. Accessed January 11, 2019. 